\title{
Asymptomatic sacral tarlov cyst
}

\author{
Julian Kammel $^{1 *}$ and Franz A. Fellner ${ }^{1,2}$ \\ ${ }^{1}$ Central Radiology Institute, Kepler University Hospital, Medical Faculty of the Johannes Kepler University, Linz, Austria \\ ${ }^{2}$ Medical Faculty of the Friedrich-Alexander-University of Erlangen-Nürnberg, Erlangen, Germany
}

\section{Introduction}

Tarlov cysts are found - in varying sizes - in up to $10.6 \%$ of the population, with women being more frequently affected than men $[1,2]$. These perineural cysts, which were first described in 1938 in the context of autopsies [3], are dilatations of nerve root pockets filled with liquor [3,4] and typically without symptoms [2]. According to Nabor et al. they are classified as spinal meningeal cysts type II due to their extradural location with neural / neural structures included [5]. MR imaging typically shows them to be hypointense in T1-weighted sequences and with high signal intensity in T2-weighted sequences [3]. A significant uptake of contrast agent was not observed.

\section{Case report}

A contrast-enhanced abdomen CT examination of a 20-year-old woman in good general condition with primarily unclear abdominalgia revealed the incidental finding of a large cystic sacral spinal mass. Clinically, there were no neurological symptoms or a neurological history. The abdominal CT also revealed acute appendicitis; This was followed by a laparoscopic appendectomy. The postoperative followup was inconspicuous. In an additioinal MR imaging examination for a more precise evaluation of the soft tissue, the spinal meningeal cystic mass was identical in position, shape and size to the previous CT examination. This was hyperintense in the T2-weighted sequences, hypointense in the T1-weighted sequences, with partially existing fine septation and broad-based parts extending ventrally through the sacral neuroforamina (Figure 1)

The mass showed a maximum extension of cranio-caudal $96 \mathrm{~mm}$, medio-lateral $69 \mathrm{~mm}$, and anterior-posterior $36 \mathrm{~mm}$ (Figure 2).

The adjacent vertebral bodies L5 to S4 as well as the corresponding vertebral arches were partially thinned without cortical interruption. Most of the spinal nerves were pushed to the edge of the spinal canal and neuroforamina. Especially in the cranial cyst area there were also intracystic neural structures. No significant enhancement was found after application of the contrast medium (Figure 3).

With extradural cyst localisation - according to Nabor et al. - the mass corresponded primarily to a spinal meningeal cyst type II (Tarlov cyst).

\section{Discussion}

Tarlov cysts are relatively common incidental findings in computed tomography and magnetic resonance imaging examinations of other parts of the body. They are mostly clinically silent [1,3]. Clinical information, including a careful medical history, naturally play a significant role in the correct evaluation of these random radiological

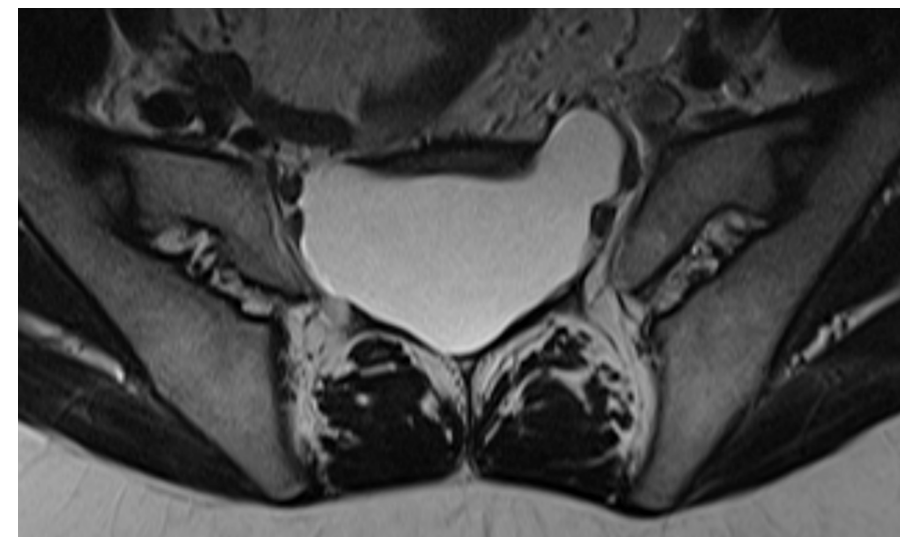

Figure 1. T2-weighted turbo spin-echo image in transverse orientation: Ventrally spreading cystic structures
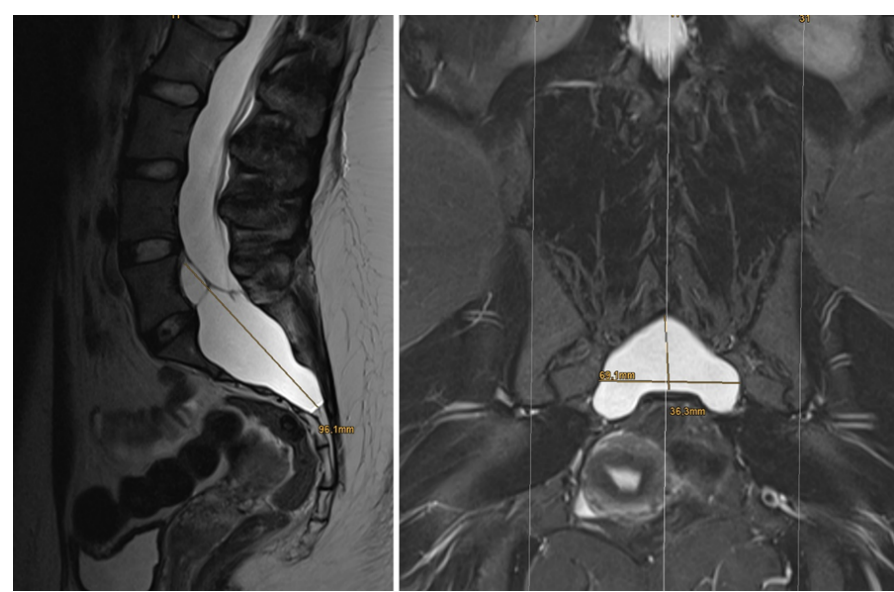

Figure 2. The axial and sagittal T2-weighted turbo spin echo images show clearly the extent of the Tarlov cyst in these two planes

${ }^{*}$ Correspondence to: Julian Kammel, Central Radiology Institute, Kepler University Hospital, Medical Faculty of the Johannes Kepler University, Linz, Austria, E-mail: Julian.Kammel2@kepleruniklinikum.at

Key words: perineural cyst, tarlov cyst, sacral mass, magnetic resonance (MR) imaging

Received: October 03, 2020; Accepted: October 19, 2020; Published: October 22,2020 


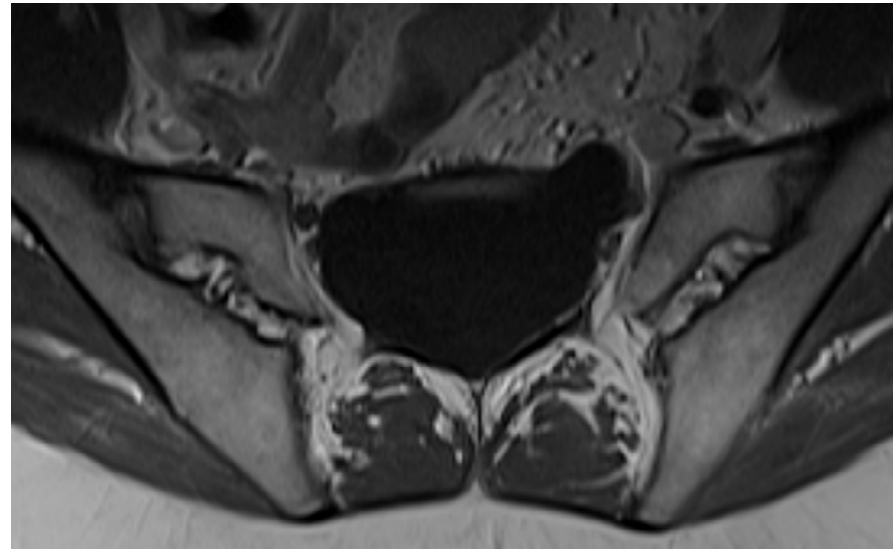

Figure 3. Axial T1-weighted image after i.v. application of contrast agent (Gadovist)

findings [6]. Both CT and MR imaging are ideally suited for the reliable detection of such Tarlov cysts [3]. The case presented here shows the advantages of magnetic resonance imaging in the exact representation of the positional relationships, especially the relationship to the adjacent nerve roots.
This case shows how even extensive cystic spinal extradural masses with nerve root reference can remain clinically asymptomatic and that in such cases appropriate care is required in assessment and diagnosis. MR tomography is far superior to $\mathrm{CT}$ in visualizing the lesion and assessing the situation.

\section{Conflicts of interest}

The authors declare that they have no conflicts of interest.

\section{References}

1. Burdan F, Mocarska A, Janczarek M, Klepacz R, Łosicki M, et al. (2013) Incidence of spinal perineurial (Tarlov) cysts among East-European patients. PLoS One 1; 8: e71514.

2. Paulsen RD, Call GA, Murtagh FR (1994) Prevalence and percutaneous drainage of cysts of the sacral nerve root sheath (Tarlov cysts). AJNR Am J Neuroradiol 15: 293297.

3. Andrieux C, Poglia P, Laudato P (2017) Tarlov Cyst: A diagnostic of exclusion. Int J Surg Case Rep 39: 25-28.

4. Tarlov IM (1938) Perineurial cysts oft he spinal nerve roots. Arch NeurPsych 40: 1067 1074

5. Nabors MW, Pait TG, Byrd EB, Karim NO, Davis DO, et al. (1988) Updated assessment and current classification of spinal meningeal cysts. J Neurosurg 68: 366-377.

6. Lucantoni C, Than KD, Wang AC, Valdivia-Valdivia JM, Maher CO, et al. (2011) Tarlov cysts: a controversial lesion of the sacral spine. Neurosurg Focus 31: E14.

Copyright: (C2020 Kammel J. This is an open-access article distributed under the terms of the Creative Commons Attribution License, which permits unrestricted use, distribution, and reproduction in any medium, provided the original author and source are credited. 